\title{
Prostatectomía radical. Revisión de nuestra serie en el periodo 1997-2003
}

\author{
F. Aguiló Lucia, J.F. Suárez Novo, J. Planes Morín, E. Condom Mundó* \\ Servicio de Urología. *Servicio de Anatomía Patológica. Hospital Universitario de Bellvitge. \\ Hospitalet de Llobregat. Barcelona. \\ Actas Urol Esp 2005; 29 (6): 542-549
}

\section{RESUMEN}

PROSTATECTOMÍA RADICAL. REVISIÓN DE NUESTRA SERIE EN EL PERIODO 1997-2003

Objetivos: Analizar los resultados y complicaciones de nuestra serie de 398 casos de prostatectomía radical retropúbica como tratamiento electivo del cáncer de próstata órganoconfinado.

Material y metodos: Entre enero de 1997 y junio de 2003 hemos realizado un total de 398 prostatectomías radicales. La edad media fue de 63,8 años $(45,8-78,2)$, con una media de PSA al diagnóstico de $9,32 \mathrm{ng} / \mathrm{ml}(0,9-129,7)$. La media del tiempo quirúrgico es de 141,6 minutos (70-280), y la media de días de ingreso hospitalario de 6,75 días (2-37).

Resultados: El seguimiento medio de nuestra serie ha sido de 65,18 meses. Como complicaciones peroperatorias destacamos: lesión rectal $1,8 \%$, linforrea $0,3 \%$, fístula urinaria $5 \%$. Como complicaciones tardías: estenosis anastomosis uretrovesical $6 \%$.

Hemos objetivado un porcentaje de márgenes positivos del 49,1\%. No hemos tenido ningún exitus peroperatorio. La supervivencia global de la serie es del 98,5\%, la supervivencia cáncer específica del $99,75 \%$, y la supervivencia libre de enfermedad del $84,97 \%$.

Conclusiones: La prostatectomía radical es una excelente opción de tratamiento en pacientes con cáncer de próstata órgano confinado. Es indispensable para obtener buenos resultados una correcta selección de los pacientes candidatos a tratamiento quirúrgico.

Palabras clave: Cáncer de próstata. Cirugía. Prostatectomía radical.

\section{ABSTRACT}

RADICAL PROSTATECTOMY. A REVIEW OF OUR SERIE BETWEEN 1997-2003

Objetive: To evaluate the complications and results of our series of 398 radical retropubic prostatectomies as an elective treatment for clinically localized prostate cancer.

Patients and methods: Between january 1997 and june 2003, a total of 398 radical retropubic prostatectomies have been performed. Mean age was 63.8 years (45.8-78.2), mean PSA at diagnosis 9.32 $\mathrm{ng} / \mathrm{ml}$ (0.9-129.7). Mean surgical time was 141.6 minutes (70-280), and mean hospitalization was 6.75 days (2-37).

Results: Mean follow-up was 65.18 months. We report as peroperatory complications: rectal injury $1.8 \%$, lymphatic leakage $0.3 \%$, urinary fistula $5 \%$. As delay complications: uretrovesical junction stenosis $6 \%$. We observed $49.1 \%$ of patients with positive surgical margins. We don't report any peroperatory death. The overall survival rate is $98.5 \%$, the cancer specific survival rate is $99.75 \%$, and the recurrence-free survival rate is $84.97 \%$.

Conclusions: Radical retropubic prostatectomy is an excellent treatment form for patients with clinically localized prostate cancer. A strict selection of patients candidates is important to obtain good results. 
$\mathrm{E}$ abordaje terapéutico del cáncer de próstata órganoconfinado es motivo de constantes revisiones y discusiones en las reuniones urooncológicas dada la diversidad de opciones terapéuticas de las que disponemos hoy en día. La conducta expectante y observación, el tratamiento hormonal, la prostatectomía radical (con sus diferentes vías de abordaje), la braquiterapia y la radioterapia externa constituyen las principales armas para afrontar el tratamiento del cáncer de próstata, que representa la neoplasia no cutánea más frecuentemente diagnosticada en varones mayores de 60 años. En Estados Unidos ha desbancado del primer puesto al cáncer de pulmón gracias a las campañas antitabaco, y constituye la segunda causa de muerte por cáncer en varones en España ${ }^{1,2}$.

La progresiva disponibilidad de la determinación de PSA en los centros sanitarios así como la mayor concienciación de la población masculina sobre la conveniencia de acudir al urólogo a partir de los 50 años, ocasiona en los últimos años un aumento del diagnóstico del cáncer de próstata órganoconfinado ${ }^{3,4}$.

Nuestro hospital es centro de referencia de una población de alrededor de 1.150.000 habitantes, lo que comporta que a nuestro servicio lleguen pacientes diagnosticados de adenocarcinoma de próstata procedentes de diferentes centros de atención primaria y consecuentemente, biopsiados y anatomopatológicamente estudiados en diferentes centros y por diferentes anatomopatólogos. Desde el año 2000 y para intentar homogeneizar y así evitar sesgos en la toma de decisiones en cuanto al tratamiento se refiere, todas las biopsias de los enfermos remitidos a nuestro servicio han sido revisadas por un mismo uropatólogo de nuestro centro.

La política seguida en nuestro servicio, una vez diagnosticado el cáncer de próstata localizado, consiste en informar al paciente de las diferentes opciones terapéuticas que ofrecemos en el servicio y de la morbilidad de todas ellas (observación y conducta expectante, cirugía radical, braquiterapia o radioterapia) y tomar de forma conjunta la decisión en cuanto al tratamiento se refiere.

Presentamos nuestra serie de 398 prostatectomías radicales realizadas entre enero de 1997 y
Junio de 2003. Desde el año 2000 se lleva a cabo la preservación de bandeletas neurovasculares en aquellos pacientes menores de 65 años, sin disfunción eréctil previa al diagnóstico, que presentan en las biopsias prostáticas transrectales un Adenocarcinoma Gleason $<7$ con afectación de un solo lóbulo prostático, y finalmente PSA igual o menor a $10 \mathrm{ng} / \mathrm{ml}$.

Analizamos a continuación los resultados de nuestra serie.

\section{MATERIAL Y METODOS}

Entre enero de 1997 y junio de 2003 hemos realizado en nuestro servicio un total de 398 prostatectomías radicales en pacientes diagnosticados de Adenocarcinoma de próstata.

La edad media al diagnóstico de estos pacientes ha sido de 63.8 años (rango: 45,8-78,2). El valor medio del PSA al diagnóstico ha sido de 9,32 $\mathrm{ng} / \mathrm{ml}$ (rango: 0,90-129,70). Los valores extremos de los rangos (edad 78,2 y PSA 129,7) pertenecen a enfermos operados al inicio de la serie (año 1997). Al inicio de la serie no existía en el servicio ningún protocolo de actuación frente a enfermos diagnosticados de cáncer de próstata, y todos los miembros del "staff" realizaban la cirugía radical de próstata. Desde el año 2000 se establece la unidad de cáncer de próstata ocupándose únicamente dos miembros del "staff" médico de la misma, que son los que han realizado prácticamente la totalidad de las prostatectomías (F.A y J.F.S.). Es a partir de esa fecha cuando se limita la prostatectomía radical a aquellos pacientes con carcinoma de próstata organoconfinado, con PSA inferiores a $20 \mathrm{ng} / \mathrm{ml}$ y con una expectativa de vida superior a 10 años, mejorando la selección de enfermos candidatos a cirugía radical.

El estadiaje clínico lo hemos realizado mediante tacto rectal, determinación de PSA sérico (incluyendo el cociente PSA libre/PSA total) y biopsia prostática transrectal ecodirigida. Únicamente en 10 casos el diagnóstico fue realizado tras el estudio anatomopatológico de una RTU prostática en pacientes afectos de hiperplasia benigna de próstata ( 5 pacientes Tla y 5 pacientes T1b). No realizamos estudio de extensión salvo que el valor de PSA sea superior a $20 \mathrm{ng} / \mathrm{ml}$ y/o Gleason $>7$. En estos casos el estudio lo realizamos con TAC abdominopélvico y Gammagrafia ósea. 
Desde el año 2000 todas las prostatectomías, así como las biopsias prostáticas no realizadas en nuestro centro, han sido revisadas por el mismo uropatólogo (E.C.), con el fin de homogeneizar los criterios de cara a seleccionar el mejor tratamiento posible para cada paciente.

La técnica quirúrgica empleada ha sido la descrita por Walsh ${ }^{5}$ modificada. A pesar de que diferentes trabajos demuestran que en pacientes seleccionados la linfadenectomía obturatriz carece de sentido dado el escaso porcentaje de afectación ganglionar ${ }^{6,7,10,11}$, nosotros realizamos dicha linfadenectomía de forma rutinaria dado que consideramos que no alarga significativamente el tiempo quirúrgico y las complicaciones resultantes de la misma son mínimas. Desde el año 2000 se realiza preservación de bandeletas neurovasculares en pacientes sin disfunción eréctil previa, menores de 65 años, enfermedad en un solo lóbulo, PSA $\leq 10 \mathrm{ng} / \mathrm{ml}$ y Gleason menor de 7. El tiempo medio dispensado en el acto quirúrgico en el global de la serie ha sido de 141,60 minutos (70-280). Desde el año 2000 el tiempo medio quirúrgico se ha reducido situándose en 119 minutos.

El paciente inicia una dieta liquida a las 24 horas de la cirugía y se retira el drenaje a las 48 horas de la cirugía si el débito es inferior a $40 \mathrm{cc}$ en 24 horas. Damos de alta al enfermo a las 24 horas de haber retirado el drenaje, con una media de días de ingreso de 6,75 días. La media de días de ingreso se ha reducido desde el año 2000, siendo desde entonces de 3,5 días. La sonda vesical se retira en consultas externas a los 14-21 días de la cirugía.

\section{RESULTADOS}

La distribución anual de los casos de prostatectomía radical se resume en la Figura 1.

La mayoría de enfermos de la serie presentaban en el momento del diagnóstico un estadío clínico T1c (272 pacientes); siendo el Gleason 6 el más habitual en las biopsias prostáticas (183 pacientes) (Tabla 1).

Analizando la evolución anual del estadio clínico y el grado Gleason en las biopsias prostáticas, objetivamos un aumento de los enfermos con estadío clínico Tlc con el paso de los años, en detrimento de estadíos más avanzados fruto de

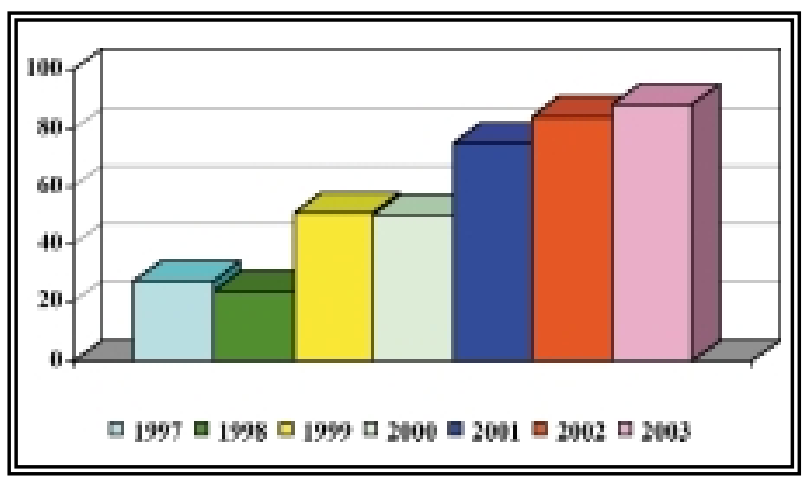

FIGURA 1. Distribución anual de casos de prostatectomia radical (Periodo comprendido entre enero de 1997 y junio de 2003).

Tabla 1

Grado Gleason de las biopsias y estadios clínicos

\begin{tabular}{cc}
\hline Estadio clinico & № casos \\
\hline T1a & 5 \\
T1b & 5 \\
T1c & 272 \\
T2a & 94 \\
T2b & 20 \\
T3a & 2 \\
\hline & \\
Gleason & o casos \\
\hline 2 & 1 \\
3 & 4 \\
4 & 35 \\
5 & 60 \\
6 & 183 \\
7 & 98 \\
8 & 12 \\
9 & 2 \\
\hline
\end{tabular}

una mejor selección de pacientes. Así, en el año 2003 todos los pacientes afectos de cáncer de próstata a los que se indicó cirugía radical presentaban un estadío clínico T1c o T2a (Fig. 2). De la misma manera, hemos objetivado al inicio de la serie una variación amplia en el grado Gleason de las biopsias, con un ligero predominio de Gleason 6 y 7 que se ha acentuado en los últimos años.

En el estudio anatomopatológico de las prostatectomías, vemos que globalmente los estadíos patológicos más frecuentes han sido el pT2b seguido del pT3a, indicando un cierto grado de infraestadiaje en el momento del diagnóstico que también refieren la mayoría de series publicadas en la literatura ${ }^{4,8,9}$. Todos nuestros casos de estadío pT4 son por afectación del cuello, criterio que 


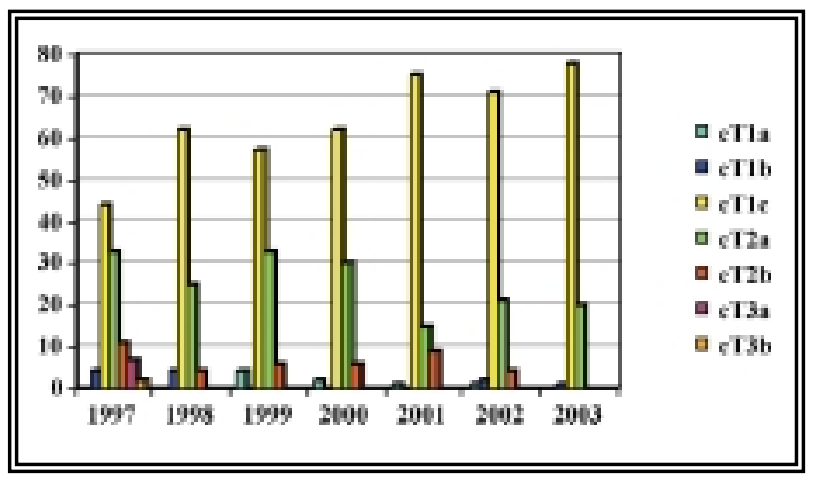

FIGURA 2. Evolución anual del estadio clinico.

cuestionamos, como otros muchos autores en comunicaciones a Congresos, de momento (Tabla 2). Al analizar la evolución anual del estadío patológico, objetivamos un cambio a partir del año 2000 disminuyendo los estadíos más avanzados, reflejo de una mejor selección de los enfermos (Fig. 3). Predomina a lo largo de toda la serie en las prostatectomías el Gleason 7, con un importante aumento del Gleason 6 en los últimos cuatro años en concordancia con un aumento del número de biopsias prostáticas con Gleason 6 en el mismo periodo (Tabla 3).

Tabla 2

Estadio patológico de las prostatectomías

\begin{tabular}{cc}
\hline Estadio patológico & № casos \\
\hline pT0 & 2 \\
pT2a & 44 \\
pT2b & 161 \\
pT3a & 123 \\
pT3b & 36 \\
pT4 & 33 \\
\hline
\end{tabular}

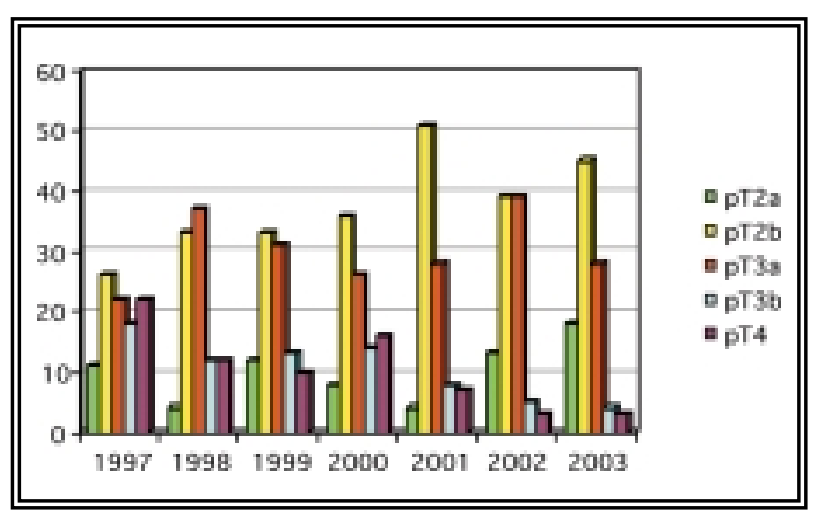

FIGURA 3. Evolución anual del estadio patológico de las prostatectomias.
Tabla 3

Grado Gleason de las prostatectomías (ND-HT: Gleason no determinable por tratamiento previo con Hormonoterapia)

\begin{tabular}{cc}
\hline Gleason & № Casos \\
\hline ND-HT & 22 \\
5 & 42 \\
6 & 96 \\
7 & 213 \\
8 & 15 \\
9 & 11 \\
\hline
\end{tabular}

El porcentaje de márgenes quirúrgicos positivos obtenido ha sido de $49,1 \%$. La distribución porcentual de márgenes quirúrgicos desde enero 1997 hasta Junio de 2003 se resume en la Figura 4. La disminución gradual del número de márgenes positivos año tras año pensamos que es debido a una mejor selección de los enfermos candidatos a cirugía y también al mayor dominio de la técnica quirúrgica de la prostatectomía radical.

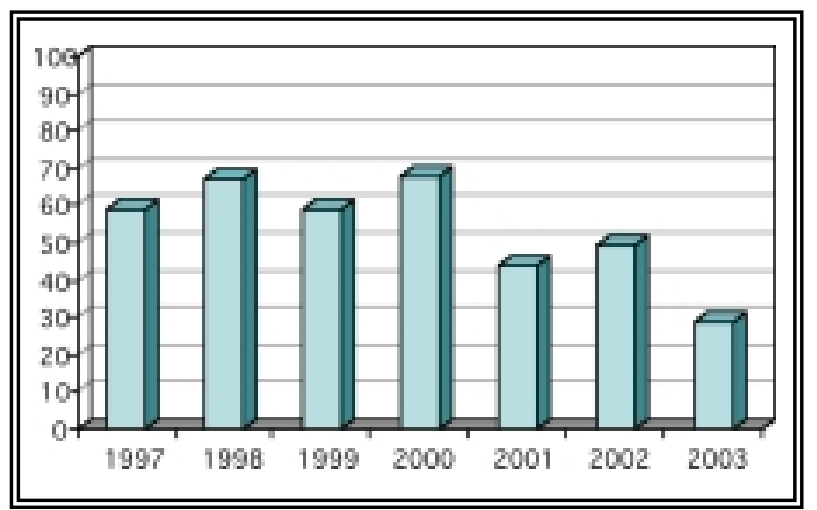

FIGURA 4. Distribución anual de márgenes positivos (porcentajes).

Si analizamos las complicaciones derivadas de la prostatectomía radical, hemos querido separarlas en peroperatorias (desde el momento de la cirugía hasta un mes de la intervención) y postoperatorias (a partir del mes de la cirugia).

Como complicaciones peroperatorias hemos tenido:

- 7 casos de lesión rectal $(1,8 \%)$, seis de ellos solventados con sutura primaria de la lesión y con dilatación anal, manteniendo al enfermo en dieta absoluta durante 4 días e iniciando posteriormente una dieta sin residuos. Sólo en un caso la lesión rectal pasó desapercibida, presentando el paciente a la semana de la intervención una 
fístula intestinal que obligó a la realización de una colostomía lateral de descarga que se cerró a los tres meses sin otras incidencias.

- En 20 pacientes (5\%) hemos objetivado fistula urinaria en la anastomosis, resolviéndose el cuadro clínico con actitud conservadora manteniendo la sonda vesical hasta la desaparición de la fuga.

- La linforrea solamente apareció en un paciente durante el postoperatorio, y se resolvió la misma de forma espontánea.

- La tasa de transfusión global de la serie (paciente que requiere la transfusión de 1 o más concentrados de hematíes en el momento de la cirugía o en los días posteriores) es del $36,1 \%$.

- No hemos tenido ningún exitus peroperatorio.

En la Tabla 4 se describen las complicaciones postquirúrgicas (lesión rectal, fístula urinaria y estenosis de anastomosis uretrovesical) desde enero 1997 hasta junio 2003.

En cuanto a las complicaciones postoperatorias o tardías, hemos analizado la estenosis de la anastomosis uretrovesical, la incontinencia urinaria y la disfunción eréctil.

- La estenosis de la anastomosis uretrovesical se ha producido en 21 pacientes $(5,3 \%$ de los casos) corrigiéndose mediante uretrotomía interna tipo Sachse.

- Para valorar la incontinencia, la hemos dividido en: continencia total, incontinencia a grandes esfuerzos (los enfermos no requieren compresas o pañales), incontinencia a medianos esfuerzos (el enfermo utiliza 1-2 pañales al día), e incontinencia a pequeños esfuerzos (3 o más pañales al día). A los tres meses de la cirugía el 87,9\% de los pacientes presentaban una buena continencia o incontinencia a grandes esfuerzos. Al año de la intervención, el porcentaje de enfermos con incontinencia a grandes esfuerzos o continencia total era del 97,1\%.
- La disfunción eréctil la hemos valorado a los tres meses y al año de la cirugía. A los tres meses el $95,4 \%$ de los pacientes presentan disfunción eréctil, mientras que al año el porcentaje disminuye levemente hasta colocarse en el 89,4\%. Reconocemos la existencia de un sesgo en la valoración de la disfunción eréctil puesto que en el período 1997-2000 no se recogía información sobre qué pacientes presentaban disfunción eréctil previa a la cirugía. Este dato se documenta en la historia clínica del paciente desde el año 2000.

Hemos llevado a cabo un análisis de supervivencia mediante el método de Kaplan-Meier. En la Figura 5 podemos objetivar la supervivencia actuarial libre de enfermedad global de la serie que es del $60 \%$ a los 80 meses. En el caso de enfermos con márgenes positivos, la supervivencia desciende al 52\% a los 80 meses, mientras que en los enfermos con márgenes negativos se sitúa en el $80 \%$ a los 80 meses (log rang test p<0,01) (Fig. 6). La supervivencia cáncer-específica es del 99,75\%, habiendo fallecido únicamente un enfermo por progresión de su cáncer de próstata.

\section{DISCUSIÓN}

Uno de los aspectos básicos que hemos objetivado al analizar los resultados de la serie y que pensamos que se debería extender a otros centros cómo el nuestro (centros de referencia con un gran volumen de enfermos procedentes de varias zonas) es el de poder contar con un uropátologo encargado de revisar las biopsias prostáticas no realizadas en el centro, así cómo de estudiar la totalidad de las piezas de prostatectomía. Desde el año 2000 así lo hemos hecho, y hemos apreciado una mayor homogeneización de los resultados del Gleason de las biopsias prostáticas y de las piezas de prostatectomía que no

Tabla 4

Evolución anual de las complicaciones quirúrgicas

\begin{tabular}{|c|c|c|c|c|c|c|c|}
\hline & 1997 & 1998 & 1999 & 2000 & 2001 & 2002 & 2003 \\
\hline Lesión rectal & $0 \%$ & $0 \%$ & $0 \%$ & $0 \%$ & $4 \%$ & $1,2 \%$ & $3,4 \%$ \\
\hline Fístula urinaria & $7,4 \%$ & $4,2 \%$ & $3,9 \%$ & $4 \%$ & $5,3 \%$ & $6 \%$ & $4,5 \%$ \\
\hline $\begin{array}{l}\text { Estenosis } \\
\text { Anastomosis }\end{array}$ & $11,1 \%$ & $8,3 \%$ & $3,9 \%$ & $2 \%$ & $6,7 \%$ & $7,1 \%$ & $2,3 \%$ \\
\hline Número prostatectomías & 27 & 24 & 51 & 50 & 75 & 84 & 88 \\
\hline
\end{tabular}




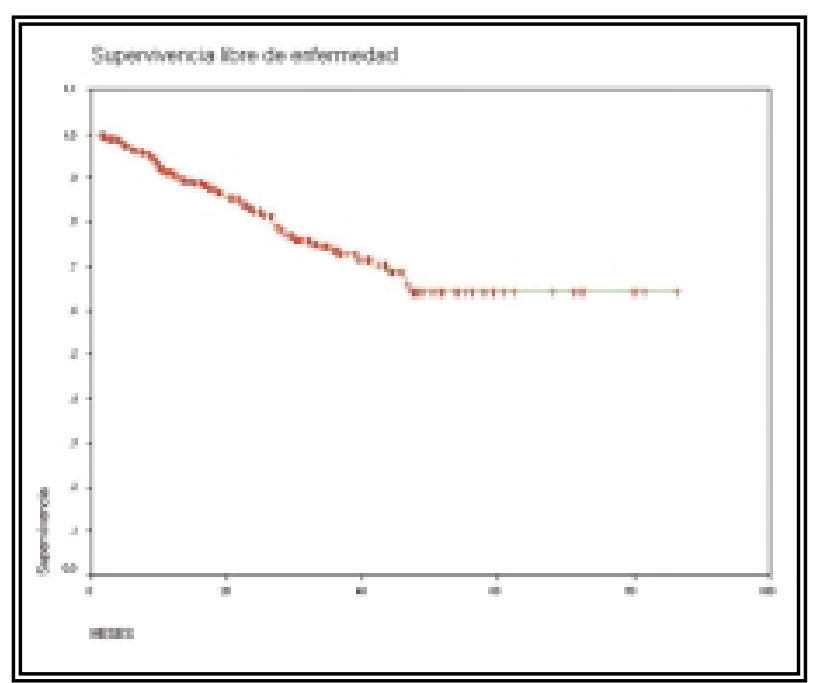

FIGURA 5. Supervivencia actuarial libre de enfermedad global de la serie.

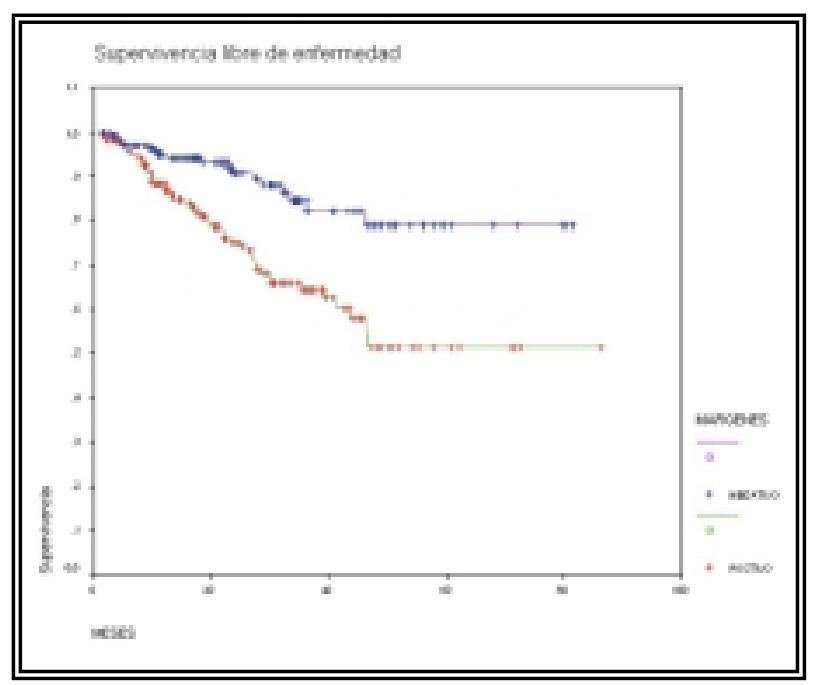

FIGURA 6. Curvas comparativas de supervivencia actuarial libre de enfermedad en enfermos con márgenes positivos y enfermos con márgenes negativos (log rank test $p<0,01$. porcentaje de enfermos incontinentes y el grado de incontinencia disminuye con el paso del tiem$\mathrm{po}^{4,9}$, sin observarse cambios pasados unos 1215 meses tras la cirugía. En nuestra serie el $97,1 \%$ de los pacientes están totalmente continentes o con una incontinencia a grandes esfuerzos (sin necesidad de protección). Consideramos que este alto porcentaje de enfermos con buena continencia es debido a la meticulosa disección y preservación del cuello vesical que realizamos durante la cirugía, y en menor medida (puesto que se realiza des del año 2000 y en pacientes seleccionados) a la preservación de las bandeletas neurovasculares.

En la Tabla 5 se comparan los porcentajes de incontinencia de diferentes series ${ }^{4,8,12,15}$.

Como hemos comentado anteriormente, nuestro grupo realiza la linfadenectomia obturatriz bilateral de forma rutinaria. Únicamente un enfermo de la serie analizada (1/398, que supone un $0,3 \%$ ) ha presentado linforrea en el postoperatorio. Probablemente esta mínima incidencia de linforrea que hemos obtenido se deba a una meticulosa disección de la fosa obturatriz ligando y clipando todas aquellas estructuras de aspecto linfático por pequeñas que sean. La baja incidencia de linforrea que hemos objetivado nos anima a seguir realizando la linfadenectomia obturatriz bilateral de forma rutinaria aprovechando la información que sobre el estadiaje nos aporta.

Hemos tenido un $1.8 \%$ de pacientes con lesión rectal, porcentaje muy similar al resto de series revisadas $4,6,8,12,16$. Como hemos mencionado anteriormente, sólo en un caso la lesión pasó desaper- observamos en el periodo 19971999. Creemos que la valoración de todas las biopsias prostáticas por un mismo uropatólogo elimina sesgos y nos ayuda a realizar una mejor selección de pacientes candidatos a cirugía radical.

Probablemente la complicación más temida de la prostatectomía radical es la incontinencia urinaria. Diferentes estudios han observado que el
Tabla 5

Comparativa de la incidencia de Incontinencia urinaria entre diferentes series (IGE: Incontinencia a grandes esfuerzos, IME: Incontinencia a medianos esfuerzos, IPE: Incontinencia a pequeños esfuerzos)

\begin{tabular}{lccccc}
\hline Autores & No casos & Continentes & I.G.E. & I.M.E. & I.P.E. \\
\hline Zincke, 1994 & 1143 & $83,5 \%$ & $9 \%$ & $4,6 \%$ & $2,9 \%$ \\
Rioja, 1997 & 204 & $56,9 \%$ & $25,1 \%$ & $9,7 \%$ & $4,6 \%$ \\
Stanford, 2000 & 1042 & $31,9 \%$ & $40,2 \%$ & $6,8 \%$ & $1,6 \%$ \\
Valdecilla, 2000 & 133 & $73 \%$ & $16,5 \%$ & $6 \%$ & $4,5 \%$ \\
Maffezzini, 2001 & 300 & $88,8 \%$ & $8,8 \%$ & & $2,3 \%$ \\
HUB, 2003 & 398 & $76 \%$ & $21,2 \%$ & $2,4 \%$ & $0,5 \%$ \\
\hline
\end{tabular}


cibida manifestándose la fístula intestinal a la semana de la intervención y requiriendo el paciente la realización de una colostomía de descarga con posterior reanastomosis a los tres meses. En el resto de casos, se realizó sutura primaria de la lesión en un plano con sutura irreabsorbible con posterior dilatación forzada anal. Se dejó a los pacientes durante cuatro días en dieta absoluta para iniciar posteriormente una dieta sin residuos.

Un 5,1\% de pacientes presentaron fuga por anastomosis. Ninguno de estos pacientes fue reintervenido por este motivo. En todos ellos se adoptó una actitud expectante manteniendo la sonda vesical hasta la resolución espontánea de la fuga.

En cuanto a complicaciones tardías, en la literatura la frecuencia de estenosis de anastomosis oscila entre $0,5 \%$ y $17,5 \%{ }^{2}$. Nosotros hemos obtenido un 5,3\% de pacientes que han presentado estenosis de la anastomosis uretrovesical. Estos casos se han resuelto mediante uretrocistoscopia y uretrotomia interna tipo Sachse asociando en ocasiones resección transuretral del tejido cicatricial, que ha siempre se ha informado de ausencia de neoplasia, actuación común en todos los autores consultados. Alguno de los pacientes afectos ha presentado recidiva del cuadro requiriendo de nuevo uretrotomia. Dos pacientes con múltiples recidivas de estenosis (uno con cuatro recidivas de estenosis de anastomosis y otro con cinco recidivas) fueron tratados endoscópicamente con uretrotomía interna en cada episodio de recidiva. Ambos presentan actualmente incontinencia total habiendo rechazado la colocación de esfínter artificial.

Otra complicación o efecto tardío que conlleva la cirugía radical es la disfunción eréctil (DE). En nuestra serie hemos observado un $89,4 \%$ de pacientes afectos de DE al año de la intervención. A pesar de que algunos grupos nacionales ${ }^{8}$ han dejado de realizar preservación de bandeletas neurovasculares en aras de la radicalidad oncológica $\mathrm{y}$ dados los pobres resultados obtenidos en lo que a potencia se refiere, nosotros realizamos preservación de bandeletas neurovasculares desde el año 2000 en aquellos pacientes menores

\section{Tabla 6} mosis uretrovesical). de 65 años, que no presentan DE previa a la cirugía, con enfermedad en un solo lóbulo y con un Gleason $<7$ y un PSA $<10$. En el momento actual no disponemos de los resultados que comparen el porcentaje de DE en el grupo de enfermos en los que se han preservado las bandeletas neurovasculares y el resto de la serie, pero sí hemos objetivado una tendencia hacia una mejoría progresiva en cuanto a disfunción eréctil e incontinencia en los últimos 3 años. Necesitamos un mayor número de pacientes y un mayor tiempo de seguimiento de los mismos para poder analizar los resultados. En la Tabla 6 presentamos una tabla donde comparamos nuestra incidencia de complicaciones con las de otras series.

A nuestro criterio, llama la atención el elevado porcentaje de márgenes positivos en el global de la serie $(49,1 \%)$ si los comparamos con otros grupos (Rioja 43\%, Portillo 34\%, Maffezzini 21,3\%, Scardino $12,8 \%)$. Nuestro grupo empezó la cirugía radical para el cáncer de próstata en el año 1992, probablemente algo más tarde que la mayoría de grupos nacionales. Este hecho, junto con una inadecuada selección de los enfermos candidatos a cirugía radical en los primeros años, influye en el resultado global de márgenes positivos de la serie. Una mejor selección posterior de los pacientes ha comportado una disminución de los márgenes positivos (con la mejoría pronóstica que ello conlleva) hasta un valor que se situó en el $29,5 \%$ en el grupo de enfermos intervenidos en el año 2003 (88 pacientes), lo cual nos sitúa en unos valores aceptables y comparables a la mayoría de grupos tanto nacionales como internacionales.

Comparativa de complicaciones entre diferentes series (FU: Fuga por anastomosis, LF: Linforrea, LR: Lesión rectal, DE: Disfunción eréctil, EA: estenosis de anasto-

\begin{tabular}{lcccccc}
\hline Autores & No casos & FU & LF & LR & DE & EA \\
\hline Zincke, 1994 & 1143 & & & $1,2 \%$ & & $4,7 \%$ \\
Heinzer, 1997 & 316 & $16,1 \%$ & $12,6 \%$ & $5 \%$ & & \\
Rioja, 1997 & 204 & $10,2 \%$ & $1,47 \%$ & $0,49 \%$ & $92,7 \%$ & $7,8 \%$ \\
Stanford, 2000 & 1042 & & & & $59,9 \%$ & \\
Valdecilla, 2000 & 133 & $9 \%$ & $5,2 \%$ & $2,2 \%$ & $98 \%$ & $12 \%$ \\
Ferrandino, 2001 & 1000 & $0,5 \%$ & $0,5 \%$ & $0,5 \%$ & & $1 \%$ \\
Maffezzini, 2002 & 300 & $0,7 \%$ & $1 \%$ & $0,3 \%$ & & $0,7 \%$ \\
HUB, 2003 & 398 & $5,1 \%$ & $0,3 \%$ & $1,8 \%$ & $89,4 \%$ & $6 \%$ \\
\hline
\end{tabular}


En la Figura 7 mostramos la evolución de los márgenes positivos con relación al número de prostatectomías a lo largo de la serie.

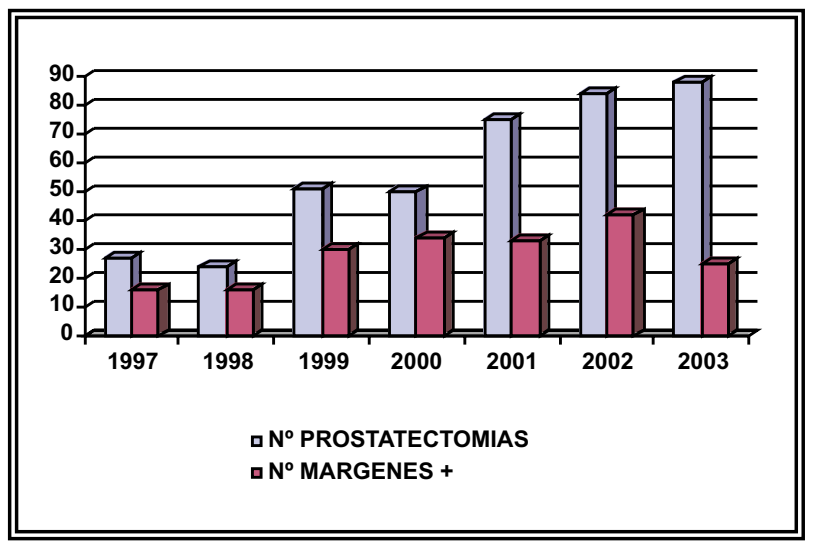

FIGURA 7. Relación anual entre número de prostatectomias y márgenes positivos.

Los resultados obtenidos en nuestra serie de 398 prostatectomías radicales en cuanto a supervivencia total y supervivencia cáncer-específica, junto a la tasa de complicaciones que hemos objetivado y que es comparable al de otras series publicadas, nos permite ratificar a la cirugía cómo uno de los tratamientos de elección para el cáncer de próstata órgano confinado. A pesar de ello, la prostatectomía comporta una morbilidad, en ocasiones no despreciable para el enfermo, y creemos que es fundamental ponerlo en conocimiento del paciente a la hora de decidir cuál va a ser el tratamiento de su enfermedad.

\section{CONCLUSIONES}

- La prostatectomía radical es un tratamiento de elección en aquellos pacientes afectos de un cáncer de próstata órganoconfinado, dados sus buenos resultados por su baja morbilidad y práctica nula mortalidad.

- Es fundamental, para la obtención de unos buenos resultados, una correcta selección de los pacientes candidatos a cirugía. Pensamos que es fundamental la revisión de las biopsias prostáticas por un único uropatólogo, con el fin de homogeneizar los criterios anatomopatológicos de selección de pacientes.

- La meticulosidad de la técnica por equipos habituados, también incide en la disminución de la morbilidad, mejorando los resultados.

\section{Agradecimientos}

Este trabajo ha sido financiado con una beca de la Agencia D“Avaluació de Tecnologia i Recerca Mèdica (AATRM) Proyecto 086/24/2000.

\section{REFERENCIAS}

1. Landis SH, Murray T, Bolden S. Cancer statistics, 1999. CA Cancer J Clin 1999;49:8-31.

2. Chiva V, Lujan M, Paez A, Santos D, Espinales G, Berenguer A. Resultados preliminares de una serie de pacientes con cáncer de próstata sometidos a prostatectomía radical. Arch Esp Urol 2004; 57(2):111-118.

3. Slawin KM, Kattan MW, Scher HI, et al. Prostate-specific antigen in screening, prognosis of the primary tumor, and monitoring of recurrent disease, in Pery MC (Ed). ASCO 2000 Educational Book. Alexandria, American Society of Clinical Oncology 2000;596-608.

4. Maffezzini M, Seveso M, Taverna G, Giusti G, Benetti A, Graziotti P. Evaluation of complications and results in a contemporary series of 300 consecutive radical retropubic prostatectomies with the anatomic approach at a single institution. Urology 2003;61:982-986.

5. Walsh PC, Lepor H, Eggleston JC. Radical prostatectomy with preservation of sexual function: anatomical and pathological considerations. Prostate 1983;4:473-485.

6. Lepor H, Nieder A, Ferrandino M. Intraoperative and postoperative complications of radical retropubic prostatectomy in a consecutive series of 1000 cases. J Urol 2001;166:1729-1733.

7. Sofer M, Hamilton-Nelson K, Civantos F, Soloway M. Positive surgical margins after radical retropubic prostatectomy: the influence of site and number on progression. J Urol 2002;167:2453-2456.

8. Rioja LA, Liedana JM, Roncales A, Gil MJ, Villanueva A, Rodriguez L, Gil P. Análisis de una serie de prostatectomías radicales. Actas Urol Esp 1997;21(9):809-816.

9. Portillo J, Rado M, Gutierrez JL, Martín B, Hernández R, Del Valle JI, Correas MA, Roca A, Hernández A. Complicaciones de una serie consecutiva de 133 casos de prostatectomía radical. Actas Urol Esp 2001;25(8):559-566.

10. Partin AW, Kattan MW, Subong EN, et al. Combination of prostate-specific antigen, clinical stage, and gleason score to predict pathological stage of localized prostate cancer. A multi-institutional update. JAMA 1997;277:1445.

11. El-Galley RE, Keane TE, Petros JA, et al. Evaluation of staging lymphadenectomy in prostate cancer. Urology 1998;52:663.

12. Zincke H, Oesterling J, Blute M, Bergstralh E, Myers R, Barrett D. Long-term (15 years) results after radical prostatectomy for clinically localized (stage T2c or lower) prostate cancer. J Urol, 1994 Nov; 152 (5 Pt 2):1850-1857.

13. Han M, Partin A, Zahurak M, Piantadosi S, Epstein J, Walsh P. Biochemical (prostate specific antigen) recurrence probability following radical prostatectomy for clinically localized prostate cancer. J Urol 2003;169:517-523.

14. Hull G, Rabbani F, Abbas F, Wheeler T, Kattan M, Scardino P. Cancer control with radical prostatectomy alone in 1000 consecutive patients. J Urol 2002;167:528-534.

15. Stanford JL, Feng Z, Hamilton AS, et al. Urinary and sexual function after radical prostatectomy for clinically localized prostate cancer. JAMA 2000:283(3):354-369.

16. Heinzer H, Graefen M, Noldus J, Hammerer P, Huland H. Early complication of anatomical radical retropubic prostatectomy: lessons from a single-center experience. Urol Intern 1997;59:30-33.

Dr. F. Aguiló Lucía

Hospital Universitari Bellvitge.

Feixa Llarga, s/n

80907 Hospitalet de Llobregat (Barcelona)

(Trabajo recibido el 30 noviembre de 2004) 